\title{
Generation of Localized Modes in an Electrical Lattice Using Subharmonic Driving
}

\author{
L. Q. English, ${ }^{1}$ F. Palmero, ${ }^{2}$ P. Candiani, ${ }^{1}$ J. Cuevas, ${ }^{2}$ R. Carretero-González, ${ }^{3}$ P. G. Kevrekidis, ${ }^{4}$ and A. J. Sievers ${ }^{5}$ \\ ${ }^{1}$ Department of Physics and Astronomy, Dickinson College, Carlisle, Pennsylvania 17013, USA \\ ${ }^{2}$ Nonlinear Physics Group, Escuela Técnica Superior de Ingeniería Informática, Departamento de Física Aplicada I, \\ Universidad de Sevilla, Avenida Reina Mercedes, s/n, 41012-Sevilla, Spain \\ ${ }^{3}$ Nonlinear Dynamical Systems Group, Department of Mathematics and Statistics, and Computational Science Research Center, \\ San Diego State University, San Diego, California 92182-7720, USA \\ ${ }^{4}$ Department of Mathematics and Statistics, University of Massachusetts, Amherst, Massachusetts 01003-4515, USA \\ ${ }^{5}$ Laboratory of Atomic and Solid State Physics, Cornell University, Ithaca, New York 14853, USA
}

(Received 10 June 2011; published 22 February 2012)

\begin{abstract}
We show experimentally and numerically that an intrinsic localized mode (ILM) can be stably produced (and experimentally observed) via subharmonic, spatially homogeneous driving in the context of a nonlinear electrical lattice. The precise nonlinear spatial response of the system has been seen to depend on the relative location in frequency between the driver frequency, $\omega_{d}$, and the bottom of the linear dispersion curve, $\omega_{0}$. If $\omega_{d} / 2$ lies just below $\omega_{0}$, then a single ILM can be generated in a 32-node lattice, whereas, when $\omega_{d} / 2$ lies within the dispersion band, a spatially extended waveform resembling a train of ILMs results. To our knowledge, and despite its apparently broad relevance, such an experimental observation of subharmonically driven ILMs has not been previously reported.
\end{abstract}

DOI: 10.1103/PhysRevLett.108.084101

PACS numbers: 05.45.Yv, 63.20.Pw, 63.20.Ry

It is well known that a damped nonlinear oscillator can respond at its intrinsic resonance frequency when it is driven at a multiple of that frequency. A direct example of such subharmonic driving is provided by the driven Van der Pol oscillator, where the ratio of response to driver frequency is exactly $1 / 3[1,2]$. Many other nonlinear oscillators exhibit similar subharmonic resonances (the Duffing oscillator being another extensively studied example). In fact, subharmonic response must be seen as a fairly generic property of nonlinear oscillators. Alternatively, a nonlinear oscillator with a parameter modulated at a particular frequency can also respond at a fraction of that frequency in what is called parametric excitation.

What happens when such nonlinear oscillators are connected to one another in a regular lattice? In nonlinear lattices, an important generic phenomenon is the existence of self-trapped localized modes, known as intrinsic localized modes (ILMs) or discrete breathers. Such a mode represents an excitation which is (typically exponentially) spatially localized over a limited range of lattice nodes and decays to zero far from these, and it is temporally periodic. In this regard, it can be thought of as an analog of the solitons of continuous media. However, the discreteness of the lattice introduces interesting variations to the problem, including, for instance, the fact that ILMs may be dynamically stable in any dimension. This has made ILMs relevant excitations for a wide array of applications, including superconducting Josephson junctions [3], photonic crystals [4], biopolymers [5], charge-transfer solids [6], antiferromagnets [7], and micromechanical cantilever arrays [8], among others [9].
Here, we blend these two broadly significant aspects of nonlinear systems by addressing the following question: can subharmonic or parametric excitations, which figure so prominently in isolated nonlinear oscillators, carry over to the lattice setting? That is, we examine whether ILMs can be generated and, especially, stabilized by subharmonic and/or parametric driving which is homogeneous in space. So far, this type of question seems to have been considered chiefly in the context of continuous media [10], or for parametric driving [11-13], and has been principally theoretical in nature. In this Letter, we demonstrate experimentally and corroborate through theoretical modeling and numerical computation, and, when possible, infusing analytical insights, that ILMs can indeed be generated and stabilized via subharmonic forcing.

The experimental system, shown in Fig. 1, is the biinductance electrical band-pass filter of Refs. [14,15], and the basic geometry and coupling to an external driver is given in Refs. [16-18]. This electrical lattice becomes nonlinear by virtue of a diode (np junction) replacing a traditional capacitor in the unit cell. The voltage at each lattice node is monitored at $0.4 \mu$ s intervals using a multichannel analog-to-digital converter. The boundary conditions are periodic, and the main result of subharmonic ILM
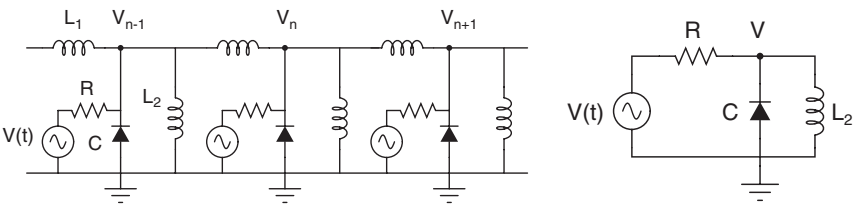

FIG. 1. Left: Schematic circuit diagram of the electrical transmission line. Right: Schematic of a single element. 
generation is realized identically in larger lattices than the one used. We use "flat" - zero voltage and current—initial conditions along the lattice. Furthermore, since the driving is homogeneous across the system, this study relates to Refs. [6-8] and more generally to nanoscale (e.g., antiferromagnets and charge-transfer solids) or even mesoscale [such as microelectromechanical system (MEMS) cantilever arrays or Josephson junctions] applications where external fields appear homogeneous on the scale of the lattice.

Using basic circuit theory, the single element composed of the parallel combination of an inductor, $L_{2}$, and a diode (driven via a resistor) is approximately described by [18]

$\frac{d v}{d \tau}=\frac{1}{c(v)}\left[\frac{\cos (\Omega \tau)}{\beta}-\frac{R+R_{l}}{\beta R_{l}} v+y-i_{D}\right]$,

$\frac{d y}{d \tau}=-\frac{1}{L_{2}} v$,

where $\beta \equiv R C_{0} \omega_{0}$ and the following dimensionless variables have been used: $\tau=\omega_{0} t ; i_{D}=I_{D} /\left(\omega_{0} C_{0} V_{d}\right) ; v=$ $V / V_{d}$, the dimensionless voltage; $c(v)=C(V) / C_{0} ; \Omega=$ $\omega_{d} / \omega_{0}$; and $\omega_{0}=1 / \sqrt{L_{2} C_{0}}$. $y$ represents the normalized current through the inductor, and $C(V)$ is the capacitance of the diode [18]. A phenomenological (and amplitudedependent) dissipation resistor, $R_{l}$, was included in the model to better approximate the experimental diode dynamics.

When $N$ such oscillators are coupled via a second inductor, Eq. (1) generalizes to the lattice equations

$$
\begin{aligned}
c\left(v_{n}\right) \frac{d v_{n}}{d \tau} & =\frac{\cos (\Omega \tau)}{\beta}-\frac{R+R_{l}}{\beta R_{l}} v_{n}+y_{n}-i_{D}\left(v_{n}\right), \\
\frac{d y_{n}}{d \tau} & =\frac{L_{2}}{L_{1}}\left(v_{n+1}+v_{n-1}-2 v_{n}\right)-v_{n} .
\end{aligned}
$$

The inductor $L_{1}$ is used to couple the unit cells, and $L_{2}$ refers to the inductor to the ground within each oscillator [18]. The ratio of these two inductors yields the effective "discreteness" of the system; in the limit of $L_{1}$ much larger (smaller) than $L_{2}$, the system can be viewed as approaching the continuum (anticontinuum) limit. In our lattice, $L_{1}=0.68 \mathrm{mH}$ and $L_{2}=0.33 \mathrm{mH}$, so that we are clearly not in the continuum limit, although the latter is, in principle, experimentally approachable and mathematically interesting in its own right.

In order to investigate the origin of these subharmonic breathers, we have to examine in detail the response of a single unit cell of the electrical lattice (i.e., an effective anticontinuum limit). As shown in Fig. 2(a), the response is a typical nonlinear resonance curve, as expected. However, for a range of frequencies located far above the linear resonance curve, the attractor of the system, which oscillates with frequency $f_{d}=\omega_{d} / 2 \pi$, experiences a perioddoubling bifurcation and a new, larger (in amplitude) attractor and appears with $f=f_{d} / 2$ (see the curve bifurcating from points $\mathrm{A}$ and $\mathrm{B}$ in the figure). Thus, for an interval
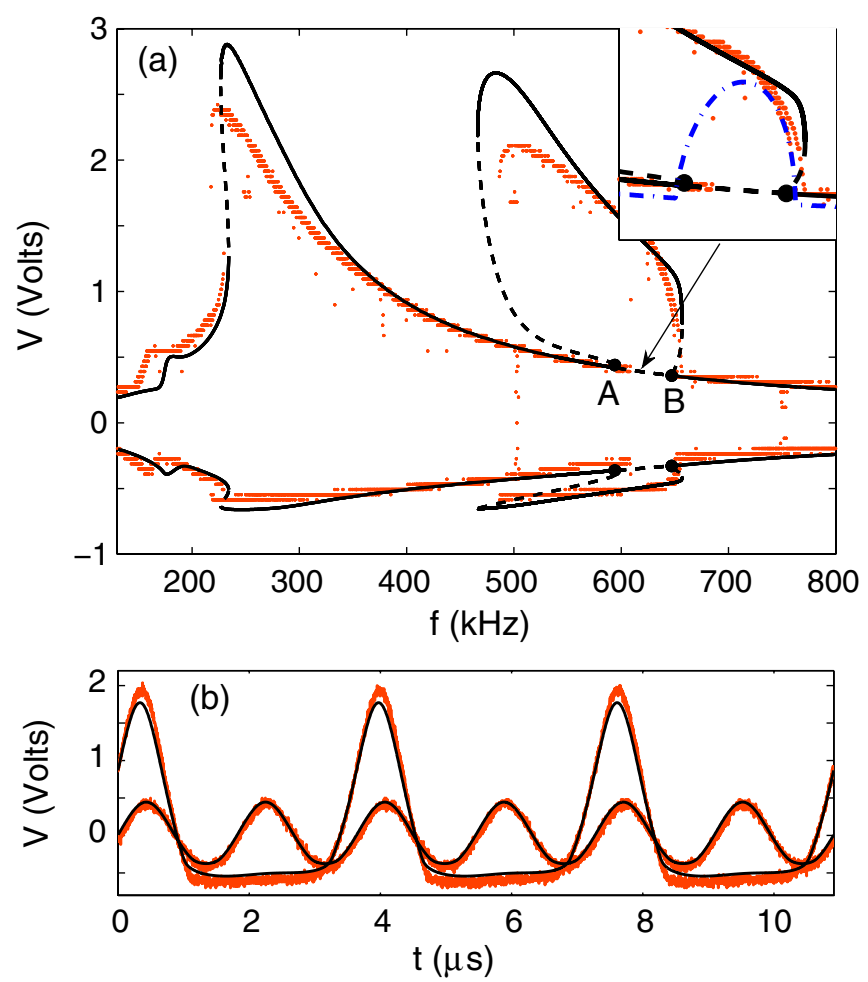

FIG. 2 (color online). Response of a unit cell at a driver amplitude of $V_{d}=8 \mathrm{~V}$ (in numerical simulations, we consider a small frequency shift of $25 \mathrm{kHz}$ to quantitatively compare with the experimental curves). Top: Nonlinear resonance curves, where grey (red) dots correspond to experimental data while the solid and dashed black lines correspond, respectively, to stable and unstable numerical solutions. Black circles show period-doubling bifurcation points. The inset zooms in on the subharmonic response, where the analytical approximation is included (dash-dotted blue line), with a frequency shift of $5 \mathrm{kHz}$. Bottom: Coexisting large and small attractors corresponding to $f_{d}=\omega_{d} / 2 \pi=550 \mathrm{kHz}$ obtained numerically (black lines) and experimentally [grey (red) lines].

of frequencies beyond the top of the linear dispersion curve of the full electrical lattice, two different attractors, one small with a frequency $f=f_{d}=550 \mathrm{kHz}$ and another one, large and with a frequency $f=f_{d} / 2=275 \mathrm{kHz}$, coexist, as illustrated in Fig. 2(b). When we decrease the voltage amplitude, $V_{d}$, the two bifurcation points (labeled A and B in the figure) get closer and, for a voltage $V_{d} \approx 6.4 \mathrm{~V}$ in the model, collide and disappear, and no subharmonic resonance takes place. Experimentally, the cutoff voltage is found around $6.2 \mathrm{~V}$; otherwise, the numerical predictions match experimental observations reasonably well, especially given the model's phenomenological treatment of the diodes.

Furthermore, in order to obtain an approximate subharmonic solution corresponding to small voltages, we can (Taylor) approximate Eq. (1) as

$$
\ddot{x}+\frac{R R_{l}}{\beta R_{l}}\left(1+x+x^{2}\right) \dot{x}+x+\frac{x^{2}}{2}=-\frac{\alpha \Omega V_{d} \sin (\Omega \tau)}{\beta},
$$


where $V=\left(x+x^{2} / 2\right) / \alpha$ (in volts) and $\alpha$ is a parameter related to nonlinear capacitance $[19,20]$. Using the harmonic balance method to approximate a solution of the subharmonic response [21], such a solution assumes the form $x(t)=A_{1} \sin (T+\phi)+A_{1 / 2} \sin (T / 2)+B_{1 / 2} \cos (T / 2)$, where $A_{1}=1 /\left(1-\omega^{2}\right)$ and $A_{1 / 2}$ and $B_{1 / 2}$ can be obtained by solving two nonlinear algebraic equations (not shown here). This approximate solution is displayed in the inset of Fig. 2(a). We note that this analytical approach predicts the range of frequencies where the subharmonic resonance takes place and the resulting solutions in the small amplitude regime.

Let us now turn to the lattice of nonlinear oscillators. Figure 3 shows the steady-state configurations upon uniform driving at frequencies (a) $f_{d}=\omega_{d} / 2 \pi=550 \mathrm{kHz}$ and (b) $f_{d}=590 \mathrm{kHz}$ and an amplitude of $7.5 \mathrm{~V}$. Note that the driver's frequency is far detuned from the system's linear eigenmodes, so that, in the linear case, we would expect no energy transfer from the driver. The uniform mode frequency $(k=0)$ at the bottom of the linear dispersion curve occurs at around $315 \mathrm{kHz}$; the top of the dispersion curve $(k=\pi)$ is at around $520 \mathrm{kHz}$.

Nevertheless, in this nonlinear system, at around $t=$ $75 \mu$ s after the driver is first turned on, energy starts to build up around the 10th node, and soon we observe a stable ILM centered there, with its wings expanding about three nodes in either direction. The ILM oscillates at its center at $275 \mathrm{kHz}$ [Fig. 3(a)] and $295 \mathrm{kHz}$ [Fig. 3(b)] but is driven at twice the corresponding frequency. It is worth mentioning that the particular location where the ILM is formed is partly due to (very slight) configurational asymmetries (i.e., very weak defects), where the driving preferentially excites a particular site of the lattice and the resulting breather state emerges spontaneously as a result of this feature. It should be mentioned that modulational instability of the (subharmonically excited) uniform mode may also arise and has been observed to give rise to multibreather states. The profiles of the ILM depicted in Fig. 3 correspond to the times at which the ILM reaches its most positive and negative voltages for both the experimental data (circles) and for the theoretical model results (solid

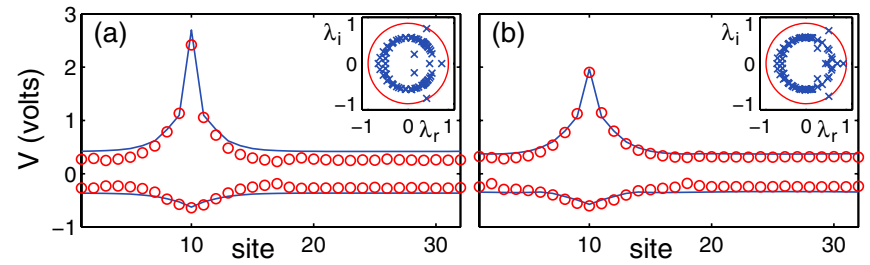

FIG. 3 (color online). Comparison between the theoretical (solid line) and experimental (circles) ILM profiles, with frequencies (a) $275 \mathrm{kHz}$ and (b) $295 \mathrm{kHz}$, generated by a homogeneous forcing of $7.5 \mathrm{~V}$ at (a) $550 \mathrm{kHz}$ and (b) $590 \mathrm{kHz}$. The insets show the Floquet multiplier numerical linearization spectrum, confirming (since all multipliers are inside the unit circle) the stability of these time-periodic solutions. line), suggesting an excellent agreement between the two approaches. The insets in (a) and (b) show the numerical linearization spectrum of Floquet multipliers $\left(\lambda=\lambda_{r}+\right.$ $i \lambda_{i}$ ) corresponding to this time-periodic solution, indicating dynamical stability of the ILM. It is interesting to note that there exists a narrow frequency interval, where the theoretical model predicts the destabilization of the ILM in favor of a stable quasiperiodic ILM through a Hopf loop (forward Hopf and reverse Hopf) bifurcation. Detailed analysis of the experimental results (frequency spectra) also reveals the corresponding window in the experiments. Further studies of this interesting bifurcation will be reported elsewhere.

Figure 4 illustrates the ILM dynamics in more detail. The top panel depicts the experimental time traces at various nodes. The most prominent trace corresponds to the ILM center; the other traces correspond to first-, second-, and third-neighbor dynamics (the experimental traces suffer from a more limited time resolution). In Fig. 4(b), the numerical traces give a smoother picture in very good agreement with the experiment; both panels demonstrate that the frequency of oscillation at the ILM's center is half of the driving frequency, $f_{d}$. Furthermore-as evidenced by the frequency spectra depicted in Figs. 4(c)4(f) - as we move away from the center to neighboring lattice nodes, a second oscillation cycle gradually appears and we transit from dominance of the $f_{d} / 2$ frequency to the eventual dominance of the fundamental frequency $f_{d}$. Thus, spatially, moving from the wings to the center, a period-doubling transition occurs.

We now explore the dependence on the driver frequency. Single-peak ILMs (shown in Fig. 3) are found between 525 and $617 \mathrm{kHz}$ (at $7.5 \mathrm{~V}$ amplitude). The lower bound is dictated by an emerging overlap with the zone-boundary linear mode, and the upper bound is dictated by the coincidence of the ILM with the uniform linear mode. As the driver frequency is raised beyond $617 \mathrm{kHz}$, the subharmonic will start to intersect the dispersion curve. What is interesting is that, even inside the linear dispersion band, localized structures can be driven subharmonically, as we will now show.
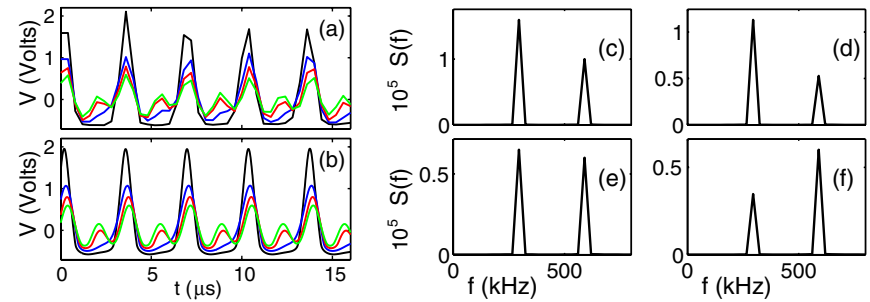

FIG. 4 (color online). (a) Experimental and (b) numerical traces of the oscillation at four different nodes - the ILM center, first neighbor, second neighbor, and third neighbor. (c)-(f) The frequency spectrum corresponding to the experimental time traces. 

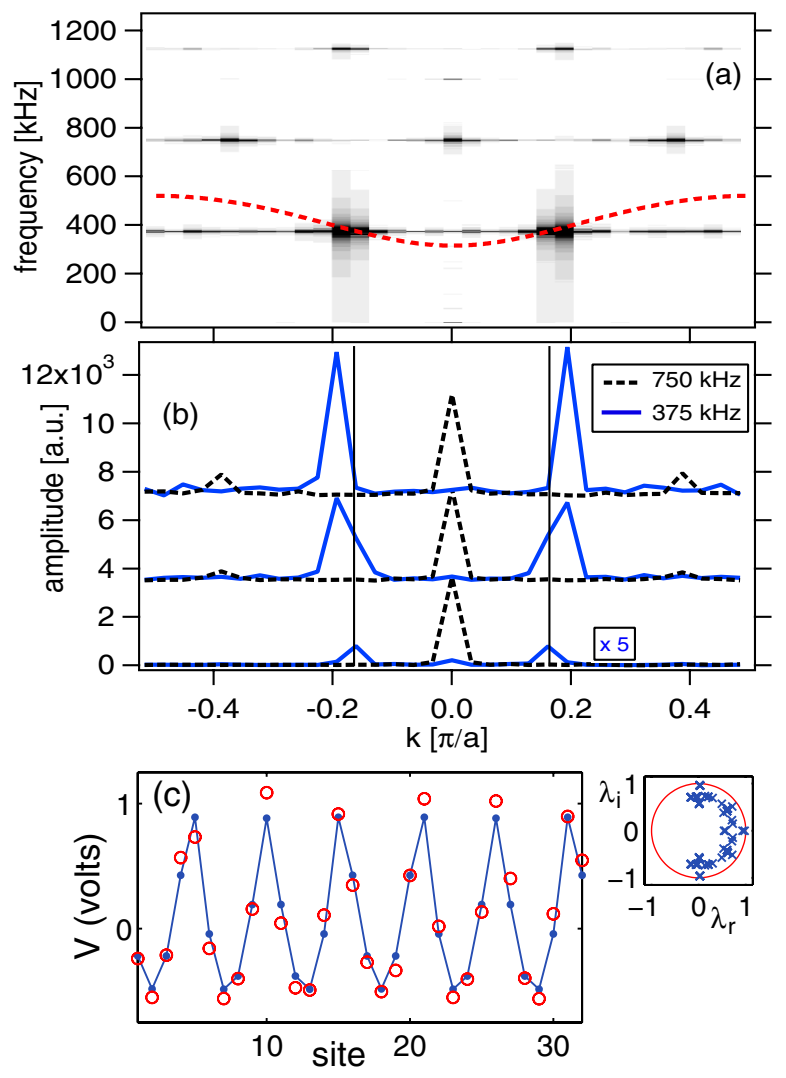

FIG. 5 (color online). (a) Two-dimensional fast Fourier transform (FFT) of the experimental lattice dynamics. (b) Spatial component of the FFT (see text for description) in (a) corresponding to $750 \mathrm{kHz}$ (solid lines) and $375 \mathrm{kHz}$ (dotted lines). (c) A spatial snapshot of the resulting spatially extended structure using the same layout as in Figs. 3(a) and 3(b).

Figure 5(a) captures the system's experimentally measured response in reciprocal space to a driver at a frequency of $750 \mathrm{kHz}$ and an amplitude of $7.92 \mathrm{~V}$. We clearly observe energy concentration at the discrete values in $k$ space where the dispersion curve (dotted red line) intersects the $f_{d} / 2$ line. Moreover, this energy concentration results from a buildup over time. Figure 5(b) plots the Fourier amplitude at $f_{d}$ and $f_{d} / 2$, for three distinct times in its evolution. The bottom trace corresponds to an early time interval, $t=$ 0 to $400 \mu \mathrm{s}$, with only a weak subharmonic response; the middle trace indicates the subharmonic response emergence, from $t=1.2 \mathrm{~ms}$ to $1.6 \mathrm{~ms}$; while the top one reveals its eventual dominance at later times, from $t=2.8 \mathrm{~ms}$ to $3.2 \mathrm{~ms}$. In the spatial domain, the pattern that results in this situation is shown in Fig. 5(c). A multipeaked localized pattern (or ILM train) is observed, the periodicity of which is set up by the wave number $k$ on the dispersion curve associated with $\omega_{d} / 2$.

Patterns resembling ILM trains, as shown in Fig. 5(c), do not appear at all driver frequencies equally. This is indicated in Fig. 6, where the linear dispersion curve (solid line) is shown with the (red) dots indicating the normal modes for a 32-node lattice. Superimposed on these linear

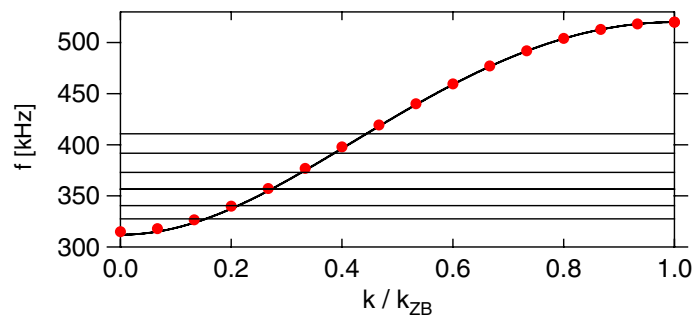

FIG. 6 (color online). Linear dispersion curve and normal modes (red dots) in relation to frequencies at which nonlinear subharmonic response is suppressed (horizontal lines) in the experiment.

normal modes are horizontal lines depicting frequencies where subharmonic response is the most difficult to accomplish experimentally. Namely, at driver frequencies equal to twice those indicated, the multipeak patterns vanish first as the amplitude of driving is reduced. These frequencies coincide well with the linear normal modes. This correlation suggests that such patterns avoid overlap with the linear spectrum and thus preferentially reside in the gaps inherent in small lattices [22,23]. Above $f=$ $420 \mathrm{kHz}\left(f_{d}>840 \mathrm{kHz}\right)$, no pattern can be induced even at the maximum driving amplitude.

In conclusion, we have demonstrated experimentally and have supported theoretically through both analysis and numerical computation the fact that the subharmonic response of coupled, driven nonlinear oscillators involves the formation of intrinsic localized modes through a spatial period-doubling sequence building up over time. We anticipate that such conclusions may have broad applicability to mechanical (pendula, granular chains), superconducting (Josephson junction), and optical systems, among others.

This research was supported by the Ministerio de Ciencia e Innovación of Spain (FIS2008-04848). A. J. S was supported by NSF-DMR-0906491. P.G.K is supported by NSF-CMMI-1000337 and the A.S. Onassis Public Benefit Foundation.

[1] F. K. Kneubühl, Oscillations and Waves (Springer-Verlag, Berlin, 1997).

[2] A.H. Nayfeh and D. T. Mook, Nonlinear Oscillations (Wiley, New York, 1979).

[3] E. Trías, J. J. Mazo, and T. P. Orlando, Phys. Rev. Lett. 84, 741 (2000); P. Binder et al., ibid. 84, 745 (2000).

[4] Yu. S. Kivshar and G. P. Agrawal, Optical Solitons: From Fibers to Photonic Crystals (Academic, San Diego, CA, 2003).

[5] A. Xie et al., Phys. Rev. Lett. 84, 5435 (2000); M. Peyrard, Nonlinearity 17, R1 (2004).

[6] B. I. Swanson et al., Phys. Rev. Lett. 82, 3288 (1999).

[7] U. T. Schwarz, L. Q. English, and A. J. Sievers, Phys. Rev. Lett. 83, 223 (1999).

[8] M. Sato et al., Rev. Mod. Phys. 78, 137 (2006). 
[9] S. Flach and A. V. Gorbach, Phys. Rep. 467, 1 (2008); S. Aubry, Physica (Amsterdam) 103D, 201 (1997).

[10] I. V. Barashenkov and Yu. S. Smirnov, Phys. Rev. E 54, 5707 (1996).

[11] E. Kenig et al., Phys. Rev. E 80, 046202 (2009).

[12] H. Susanto, Q. E. Hoq, and P. G. Kevrekidis, Phys. Rev. E 74, 067601 (2006).

[13] K. Gallo et al., Phys. Rev. Lett. 100, 053901 (2008).

[14] P. Marquie, J. M. Bilbault, and M. Remoissenet, Phys. Rev. E 49, 828 (1994).

[15] M. Remoissenet, Waves Called Solitons (Springer-Verlag, Berlin, 1993).
[16] R. Stearrett and L.Q. English, J. Phys. D 40, 5394 (2007).

[17] L. Q. English et al., Phys. Rev. E 81, 046605 (2010).

[18] F. Palmero et al., Phys. Rev. E 84, 026605 (2011).

[19] M. Sato et al., Europhys. Lett. 80, 30002 (2007).

[20] L. Q. English, R. B. Thakur, and R. Stearrett, Phys. Rev. E 77, 066601 (2008).

[21] A. A. Al-Qaisia and M. N. Hamdan, J. Sound Vib. 305, 772 (2007).

[22] A. M. Morgante et al., J. Phys. A 35, 4999 (2002).

[23] M. Sato and A. J. Sievers, Phys. Rev. Lett. 98, 214101 (2007). 